\title{
Vasculatura Portal Intrahepática: Descripción Anatómica, Radiológica y Ecográfica
}

\author{
Portal Intrahepatic Vasculature: Anatomical, Radiological and Sonographic Description
}

\author{
Gabriela Pidal,"**; Gabriela C. Oribe**; Ignacio G. Arrayago*; \\ Federico J. Curra-Gagliano*; Elena B. D’Anna** \& Esteban G. Martín***
}

PIDAL, G.; ORIBE, G. C.; ARRAYAGO, I. G.; CURRA-GAGLIANO, F. J.; D’ANNA, E. B. \& MARTíN, E. G. Vasculatura portal intrahepática: descripción anatómica, radiológica y ecográfica. Int. J. Morphol., 32(3):909-913, 2014.

RESUMEN: El presente trabajo describe la arborización del sistema venoso portal intrahepático desde los enfoques anatómico, radiológico y ecográfico. Se realizaron 5 preparados anatómicos mediante inyección de resinas plásticas y posterior corrosión. Por radiología se contrastó el árbol portal en una pieza anatómica. Se realizaron estudios ecográficos bidimensionales y Doppler en 13 caninos de raza Beagle. Estos procedimientos permitieron a los autores comparar la tridimensionalidad de los preparados anatómicos con la bidimensionalidad de los estudios por imágenes.

PALABRAS CLAVE: Canino; Circulación portal; Portografía; Ultrasonografía; Doppler.

\section{INTRODUCCIÓN}

Desde la antigüedad, el estudio descriptivo de la vasculatura hepática ocupó un lugar preponderante entre los anatomistas. Inicialmente se realizó mediante estudios post mortem de piezas anatómicas. Durante el siglo XX con el advenimiento de técnicas de diagnóstico por imágenes como la radiología con contraste endovenoso y la ultrasonografía, se corroboró lo que ya se conocía post mortem. De esta manera se pudo confeccionar un mapa vascular con la pieza in toto, y no solo por su disección en partes.

Por su parte, las técnicas de inyección intravascular con resinas plásticas y acrílicas jugaron un papel importante en la descripción y demostración de las ramificaciones vasculares en las piezas anatómicas. Estos estudios no solo constituyeron un invalorable avance en la práctica de la medicina sino también a nivel de la docencia en las disciplinas morfológicas (Ursic et al., 2007).

La ultrasonografía bidimensional y Doppler constituyen hoy en día un método de diagnóstico por imágenes fundamental en el estudio anatómico y funcional del hígado en su conjunto, permitiendo identificar afecciones y trastornos vasculares. La visualización del parénquima en tiempo real y la identificación de las estructuras vasculares portales y venosas propias, constituye un valioso aporte en la clínica diaria, delineando la segmentación hepática que permite localizar con precisión las lesiones. Por otra parte, la técnica Doppler nos permite evaluar las características de la vasculatura venosa y portal en detalle (Faverzani et al., 2006). Lo antes mencionado, hace de la ecografía el método imagenológico no invasivo de elección para la evaluación general del hígado.

El hígado se ubica en el epigastrio en relación al diafragma. Su cara parietal (diafragmática) es convexa y presenta el surco de la vena cava. Su cara visceral posee las impresiones de los órganos con los que contacta. En su parte central un área denominada Porta Hepatis constituye la entrada de la arteria hepática, vena porta, nervios autónomos, vasos linfáticos y vías de excreción biliar. La vesícula biliar se ubica en su fosa, entre el lóbulo medial derecho y cuadrado. A la izquierda de esta última y en relación al borde ventral hepático, se encuentra la fisura del ligamento redondo conteniendo a este mismo. El borde ventral del hígado se

\footnotetext{
* Unidad de Cardiología, Hospital Escuela de la Facultad de Ciencias Veterinarias, Universidad de Buenos Aires, Buenos Aires, Argentina.

** Unidad de Ecografía, Hospital Escuela de la Facultad de Ciencias Veterinarias, Universidad de Buenos Aires, Buenos Aires, Argentina.

*** Cátedra de Anatomía, Hospital Escuela de la Facultad de Ciencias Veterinarias, Universidad de Buenos Aires, Buenos Aires, Argentina. El presente manuscrito se encuentra enmarcado dentro del Proyecto de Investigación Clínica de la Secretaría de Ciencia y Técnica de la Universidad de Buenos Aires: CV-03.
} 
encuentra marcado por incisuras denominadas interlobulares que permiten la segmentación de la glándula en tres lóbulos: izquierdo, medio y derecho. El izquierdo se extiende desde el borde izquierdo hasta la fisura del ligamento redondo. El medio entre la fisura del ligamento redondo y la vesícula biliar, dividido por la porta hepatis en dos segmentos: el cuadrado en ventral y el caudado en dorsal, presentando este último dos prolongaciones, una hacia la porta hepatis, denominado proceso papilar y otra hacia la derecha denominado proceso caudado. Finalmente el lóbulo derecho se encuentra ubicado entre la vesícula biliar y el borde derecho de la glándula. Tanto el lóbulo izquierdo como el derecho se encuentran subdivididos en medial y lateral por las incisuras interlobares (Schaller, 1996).

El hígado recibe el $75 \%$ de su irrigación proveniente del sistema porta y el restante $25 \%$ de la arteria hepática, mientras que su drenaje se realiza a través de las venas hepáticas que tributan a la vena cava caudal (Molazem et al., 2007). La ecografía Doppler nos permite diferenciar entre estos tres grupos de vasos ya que cada uno posee un espectro que lo caracteriza (Spaulding, 1997).

Las venas hepáticas y su convergencia en la vena cava caudal pueden ser exploradas adecuadamente en el hígado. El número y la anatomía de las venas hepáticas se encuentran claramente descriptos (D’Anjou, 2008). Su patrón Doppler es fuertemente periódico, y es influenciado por las variaciones de presión en el atrio derecho y los movimientos del tórax durante el ciclo respiratorio (Ursic et al.; Spaulding).

El árbol portal difiere por su distribución de las venas hepáticas. El tejido fibroadiposo que forma los espacios portales conteniendo las ramas de la arteria hepática, conductos biliares y vasos linfáticos, produce ecos brillantes de la pared que ayudan a diferenciar las venas portales de las hepáticas.

\section{MATERIAL Y MÉTODO}

Anatomía . Para el siguiente trabajo se utilizaron 5 cadáveres de caninos, 1 de raza Beagle y 4 mestizos de tamaño mediano (peso promedio $16 \mathrm{~kg}$ ) excluyendo las hepatopatías como causa de deceso. A través de una incisión xifopúbica, se identificó la vena Porta principal. Esta se canalizó con una sonda $21 \mathrm{G}$ para perfundir con solución fisiológica asegurando la permeabilidad del circuito portal. Inmediatamente y por la misma vía se procedió a la inyección replesiva de este circuito venoso con látex comercial. El fraguado se realizó conservando los animales en cámara de $0^{\circ} \mathrm{C}$ durante $24 \mathrm{~h}$. Se fijó la preparación durante una semana mediante inmersión en solución acuosa de formol al 10\% y luego se procedió a la extrac- ción del hígado. El paso de fijación se obvió en las preparaciones destinadas al proceso de corrosión, el cual se cumplió a temperatura ambiente en inmersión en una solución acuosa sobresaturada de ácido sulfúrico para eliminar el parénquima hepático y poder evidenciar la vasculatura portal.

Las fotos fueron realizadas sumergiendo el preparado en un recipiente de vidrio transparente lleno de agua, con la finalidad de que la vasculatura se dispusiera de forma tridimensional similar a la situación in vivo.

Portografía. Se extrajo la pieza anatómica del cadáver de un canino hembra mestizo de 10 kilos sin evidencia clínica o de laboratorio de enfermedad hepática. Se utilizó un equipo Generay Triselex 10 de 1000 mA 150 kV y Películas Radiográficas Kodak Medical X-Ray Film General Purpose Green, de 30 x $40 \mathrm{~cm}$. Se instiló una solución de medio de contraste iodado Triyosom 50 (Meglumina diatrizoato y diatrizoico ácido) utilizando una sonda tipo Foley R8 ubicada en la vena porta principal a nivel porta hepatis. Se obtuvieron imágenes en incidencia caudo-craneal por ser las que ofrecen menos superposición de estructuras vasculares y mayor similitud con los cortes transversales realizados de epigastrio en el examen ultrasonográfico in vivo.

Ultrasonografía. Se utilizaron para este estudio 13 caninos de raza Beagle de edades variables (rango 1 a 15 años) de 9 a 14 kilogramos de peso, de ambos sexos, sin evidencia de enfermedad hepática. El procedimiento se realizó luego de un ayuno sólido de 6 horas, sin restricción de líquidos. Todos los caninos fueron explorados por medio de sujeción manual sin sedación.

Se utilizó un ecógrafo Doppler Mindray M5 VET ® con sonda microconvexa electrónica multifrecuencia (2.5 a 5 $\mathrm{MHz}$ ). Para todos los caninos, se utilizó la frecuencia de 5 MHz. Se realizó tricotomía de las paredes costales derecha y región ventral de epigastrio. Se utilizó alcohol y gel de acoplamiento acústico. Para la exploración bidimensional en blanco y negro se configuró el equipo con una frecuencia de $5 \mathrm{MHz}, 37$ cuadros por segundo, rango dinámico 75, profundidad variable de acuerdo al objeto de la exploración. Ganancia general 54.

Para la exploración Doppler espectral pulsado, se configuró un ángulo de corrección de $+60^{\circ},-60^{\circ}$ o sin ángulo $\left(0^{\circ}\right)$ según la disposición del vaso a explorar con respecto al plano de corte. El ángulo de insonación (formado por los haces de ultrasonido emitidos por el transductor y el flujo de sangre del vaso a explorar) es un factor de gran importancia a tener en cuenta durante la exploración Doppler de los vasos el mismo debería ser de $0^{\circ}$ o $180^{\circ}$, para no inducir a errores por defecto en los valores de velocidad encontrados. Tanto 
en medicina humana como en medicina veterinaria se ha establecido que dicho ángulo no debe superar los $60^{\circ}$ de manera de reducir el posible error en las mediciones (Penninck \& Finn-Bodner, 1998; Nyland et al., 2004).

La dificultad de obtener un ángulo por debajo de $60^{\circ}$ es uno de los obstáculos a sortear cuando se evalúan los vasos hepáticos, cuando alinear el flujo vascular y el haz de ultrasonido no fue posible se recurrió a la función de ángulo automático provista por el ecógrafo. La ventana de interrogación utilizada en Doppler Espectral Pulsado fue de 0,5 a $1 \mathrm{~mm}$.

Para realizar exploración Doppler Color se configuró el equipo automáticamente se disminuyó la ganancia general del equipo y la frecuencia del transductor. Para la exploración de la región porta hepatis se abordaron los espacios intercostales $9^{\circ}$ a $12^{\circ}$ y la región epigástrica desde el tercio dorsal, en ambos casos con el paciente en decúbito lateral izquierdo.

\section{RESULTADOS}

Anatomía. De los 5 preparados hepáticos que se realizaron según la técnica descripta, 4 evidenciaron con claridad las ramas de primero, segundo orden. No se pudieron poner en clara evidencia las de cuarto orden, mientras que las de tercer orden no fueron visibles en forma constante por falta de degradación de tejido adyacente o por malograrse durante la corrosión. El patrón venoso portal observado en los preparados realizados se mantuvo relativamente constante. Se observaron con claridad la vena porta principal, impar, que transporta sangre desde sus tributarias provenientes del tracto gastrointestinal, del páncreas y el bazo. Del árbol portal intrahepático se reconocieron las ramas derecha e izquierda. La rama derecha de trayecto corto, emite de una o dos subsidiarias que se dirigen dorsolateralmente hacia el proceso caudado y de una a tres ramas que ventrolateralmente se ramifican en ellóbulo lateral derecho. La rama izquierda se dirige transversalmente hacia los lóbulos medio e izquierdo y al segmento medial del lóbulo derecho. Desde su inicio la rama izquierda principal se dirige ventralmente hacia el segmento medial del lóbulo derecho, para el lóbulo medio emite una a tres ramas dorsales para el proceso papilar y una a tres ventrolaterales el lóbulo cuadrado. Finalizando en el lóbulo izquierdo con una rama para el lóbulo medial y tres ramas para el lóbulo lateral (Fig. 1).

Portografía El extremo craneal de la sonda se ubica en la rama principal de la porta izquierda, lo que genera un defecto de llenado. El medio de contraste logra una distribución uniforme del contraste hasta los vasos de $2^{\circ}$ y $3^{\circ}$ orden.

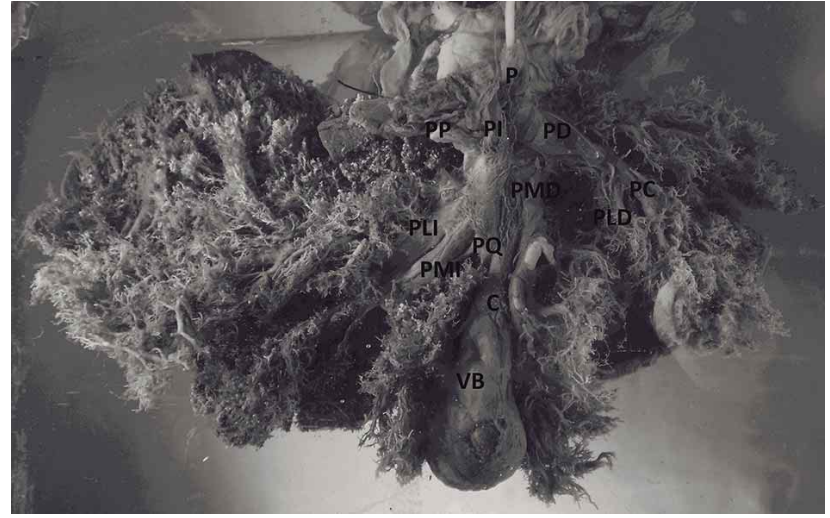

Fig. 1. Preparación anatómica inyectada con látex y posteriormente sometida a corrosión, donde se puede observar la vasculatura portal. P: porta principal, PD: porta derecha, PC: porta lóbulo caudado; PLD: porta lóbulo lateral derecho, PI: porta izquierda, PMD: porta lóbulo medial derecho, PP: porta proceso papilar, PQ: porta proceso cuadrado, PMI: porta lóbulo medial izquierdo, PLI: porta lóbulo lateral izquierdo, C: conducto cístico, VB: vesícula biliar.

El vaso principal que recorre el parénquima desde la porta hepatis hasta la extremidad izquierda en correspondencia con la mayor masa hepática, es la gran vena porta izquierda, dando ramas para el lóbulo cuadrado, para las subdivisiones (lóbulo medial y lateral izquierdo) y para el lóbulo medial derecho.

La irrigación de los lóbulos lateral derecho y caudado se vehiculiza por ramas separadas que se desprenden de la porta principal cercanas al hilio hepático.

La rama papilar de la porta que irriga este sublóbulo del caudado y que se origina de la porta izquierda, se contrasta desde la cara lateral izquierda de la porta izquierda como primera rama de calibre discreto (Fig. 2A y B).

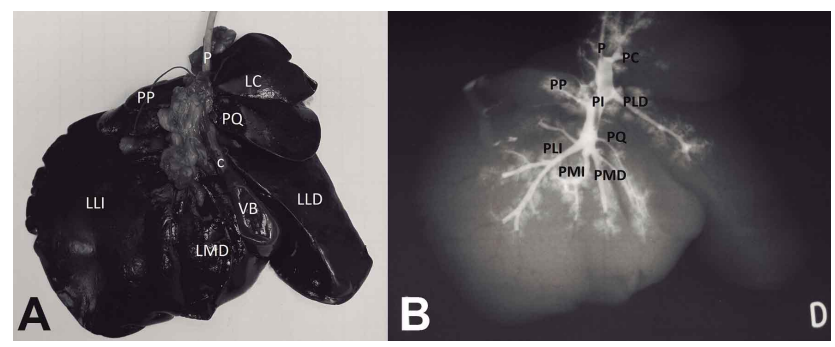

Fig. 2. A) Pieza anatómica. P: vena porta, LC: lóbulo caudado, PQ: proceso cuadrado, LLD: lóbulo lateral derecho, LMD: lóbulo medial derecho, LLI: lóbulo lateral izquierdo, PP: proceso papilar, C: conducto cístico, VB: vesícula biliar. B) Estudio portográfico, donde se observa el árbol portal, hasta los vasos de $2^{\circ}$ y $3^{\circ}$ orden. P: vena porta, PI: porta izquierda, PC: porta caudado, PLD: porta lateral derecho, PQ: porta cuadrado, PMD: porta medial derecho, PMI: porta medial izquierdo, PLI: porta lateral izquierdo, PP: porta proceso papilar. 
Ultrasonografía. La vena porta izquierda, mejor visualizada en un plano transversal desde el epigastrio, se identifica caudal y dorsal al cuello de la vesícula biliar entre ésta y las venas hepáticas. Las ramas de la vena porta izquierda se observan en las porciones lateral y medial izquierdas en relación ventral a la vesícula biliar. La vena porta lateral izquierda presenta mayor recorrido dentro del parénquima que su segmento medial izquierdo y esto es acorde al territorio que irriga. La vena porta del lóbulo cuadrado surge del tronco principal o de las ramas de la vena porta medial izquierda. Esto contrasta con el sistema venoso hepático, en el cual la vena hepática del lóbulo cuadrado normalmente se une con la vena hepática medial derecha. La vena porta medial derecha surge del tronco principal para entrar en el lóbulo homónimo, a la derecha de la vesícula biliar (Fig. 3). Las ramas de la vena porta hacia los lóbulos lateral derecho y caudado son más difíciles de identificar y no pueden visualizarse en el mismo plano que la vena porta izquierda. Estas ramas pueden explorarse tanto desde el abdomen ventral como desde una aproximación intercostal derecha a través de los espacios novenos a undécimo. Los vasos portales lateral derecho y caudado normalmente surgen juntos a partir de la vena porta (Fig. 4).

El patrón de espectros obtenidos fue continuo, monofásico, de baja resistencia, hepatópeto (flujo que ingresa al parénquima). En la mayoría de los registros el espectro fue positivo, inscribiéndose por encima de la línea de base en el Doppler espectral y en tonos de rojo intenso en el Doppler color (Fig. 5). En pocos registros, principalmente al interrogar algún segmento de la vena porta izquierda, se obtuvieron señales de flujo negativo y color azul. En cuanto a la velocidad máxima se encontraron sutiles ondulaciones en su registro.

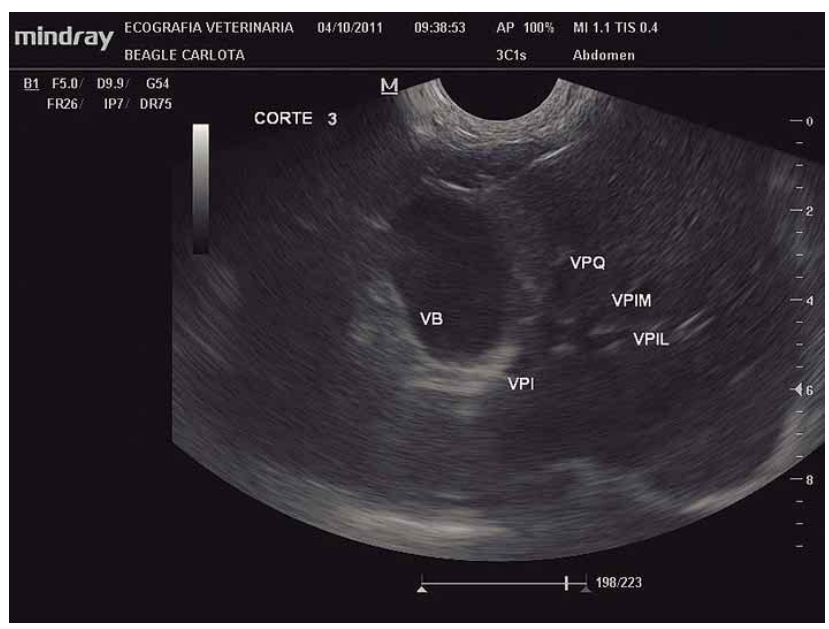

Fig. 3. Imagen ecográfica bidimensional hepática en un plano transversal desde el epigastrio. Se identifican la vesícula biliar (VB), la vena porta izquierda (VPI) y sus ramas para el lóbulo cuadrado (VPQ), medial izquierdo (VPM) y lateral izquierdo (VPL).

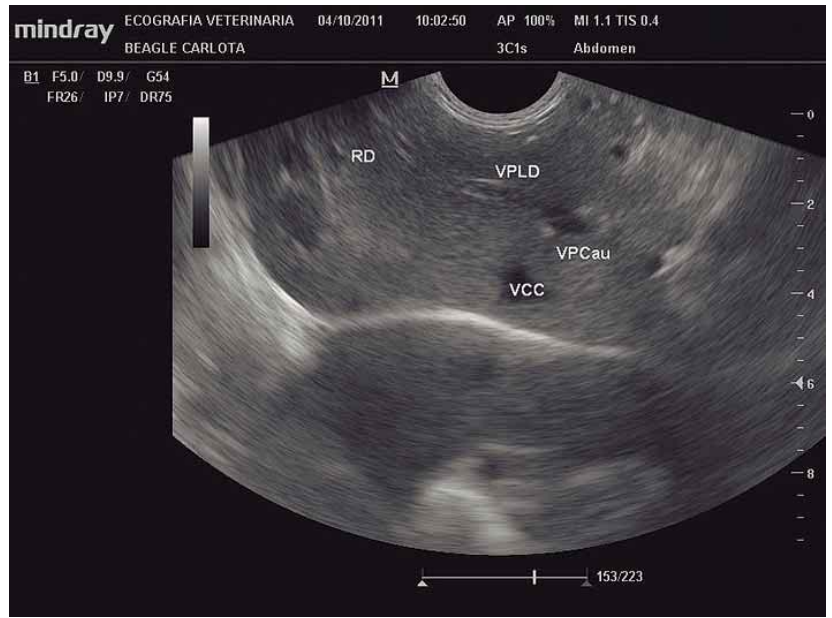

Fig. 4. Imagen ecográfica bidimensional hepática registrada desde una aproximación intercostal derecha a través de los espacios novenos a undécimo. Se visualizan la vena porta lateral derecha (VPLD), la vena porta del lóbulo caudado (VPCau), la vena cava caudal en un corte transversal (VCC) y un corte oblicuo del riñón derecho (RD).

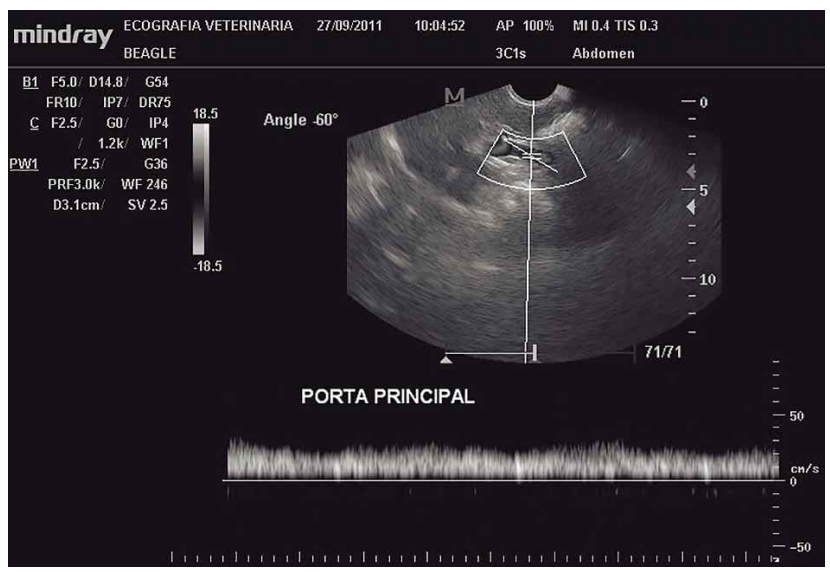

Fig. 5. Imagen triplex donde se observa arriba el parénquima hepático y la vena porta (a la cual se explora mediante Doppler color) en un plano longitudinal desde una aproximación lateral derecha a nivel del undécimo espacio intercostal derecho. Abajo espectro positivo y homogéneo característico de la vena porta (velocidad entre 20 y $30 \mathrm{~cm} / \mathrm{seg}$ ).

\section{DISCUSIÓN}

En el campo morfológico, las técnicas de inyección intravascular con resinas plásticas y acrílicas permiten crear un preparado tridimensional que facilita la aprehensión del conocimiento del árbol portal, fundamental para interpretar el producto bidimensional de los métodos de diagnóstico por imágenes.

Los protocolos radiológicos con contraste del árbol portal, indicados para evaluar posibles anastomosis portosistémicas, son: la esplenoportografía transabdominal e 
intraquirúrgica y la angiografía de la arteria mesentérica craneal (O`Brien, 1978).

De todas las técnicas mencionadas se obtienen imágenes en incidencia látero-lateral con la consecuente superposición de las ramas intrahepáticas. A diferencia de las pesquisas clínicas el objetivo del presente trabajo fue describir la anatomía del árbol portal. Para ello se contrastó la vasculatura en la pieza anatómica, presentando como principal ventaja la obtención de imágenes caudo- craneales que permite visualizar el árbol portal de manera similar a los cortes ecográficos transversales, evitando la superposición que se produce en las incidencias laterales in vivo.

La veno-portografía mesentérica directa es un método invasivo que requiere intervención quirúrgica a cielo abierto, incrementando riesgos para el paciente, restringiendo su realización a centros de alta complejidad. La esplenoportografía es una alternativa a cielo cerrado, realizable con guía ecográfica, no exenta de riesgos como hematomas o ruptura capsular esplénica (Wrigley, 1985).

En contraste con los protocolos radiológicos, la ultrasonografía, es un método de alta disponibilidad, y nula invasividad, que con el entrenamiento adecuado permite identificar en forma precisa la vascultura hepática e inferir mediante ésta la localización anatómica de las lesiones.

En la exploración en modalidad Doppler del tronco principal y ramas de primer orden se logró un trazado continuo, mientras que en las ramas de tercer orden generalmente los registros fueron discontinuos. Estos fenómenos ocurren debido al desplazamiento del parénquima hepático generado por los movimientos ventilatorios, que desplazan en forma rítmica y coincidente el volumen de muestra, siendo más evidente en la espiración.

La ecografía bidimensional y Doppler constituyen hoy en día un método de diagnóstico por imágenes fundamental en el estudio anatómico y funcional del hígado en su conjunto, permitiendo identificar afecciones y trastornos vasculares. La visualización del parénquima en tiempo real y la identificación de las estructuras vasculares portales y venosas propias, constituye un valioso aporte en la clínica diaria, delineando la segmentación hepática permitiendo la localización de las lesiones.

PIDAL, G.; ORIBE, G. C.; ARRAYAGO, I. G.; CURRAGAGLiANO, F. J.; D'ANNA, E. B. \& MARTín, E. G. Portal intrahepatic vasculature: anatomical, radiological and sonographic description. Int. J. Morphol., 32(3):909-913, 2014.

SUMMARY: This article describes the distribution of the intrahepatic portal vein system by means of anatomy, radiology and ultrasound echo-scan approach. Five anatomical resin-embedded vein preparations with posterior corrosion have been done. The portal tree was depicted by radiology. Ultrasound B-mode and Doppler Scans were performed on 13 beagle dogs. These procedures allowed the authors to compare the threedimensional preparations with the two-dimensional imaging methods.

KEY WORDS: Canine; Portal vein anatomy; Portography; Ultrasonography; Doppler.

\section{REFERENCIAS BIBLIOGRÁFICAS}

D’Anjou, M. A. Chapter six: Liver. In: Penninck, D. \& D'Anjou, M. A. (Eds.). Atlas of Small Animal Ultrasonography. Ames, Blackwell Publishing, 2008.

Faverzani, S.; Chinosi, S. \& Lodi, M. The role of hepatic ultrasonography in small animal medicine: from the identification of the lesions to diagnosis. Vet. Res. Commun., 30(Suppl. 1):139-43, 2006.

Molazem, M.; Vajhi, A.; Soroori, S.; Veshkini, A.; Masoudifard, M. \& Pedram, S. Three-dimensional color Doppler ultrasonography study of normal liver vascular pattern in dog. Iran. J. Vet. Surg., 2(3):4957, 2007.

Nyland, T. G.; Matoon, J. S.; Herrgesell, E. J. \& Wisner, E. Hígado. En: Nyland, T. G. \& Matoon, J. S. Diagnóstico ecográfico en pequeños animales. 2a ed. Barcelona, Saunders Company, 2004.

O`Brien, T. R. Radiographic diagnosis of abdominal disorders in dog and cat. Philadelphia, W. B. Saunders Company, 1978.

Penninck, D. G. \& Finn-Bodner, S. T. Updates in interventional ultrasonography. Vet. Clin. North Am. Small Anim. Pract., 28(4):101740, 1998.

Schaller, O. L. B. S. Nomenclatura Anatómica Veterinaria Ilustrada. Zaragoza, Acribia Editorial, 1996.

Spaulding, K. A. A review of sonographic identification of abdominal blood vessels and juxtavascular organs. Vet. Radiol. Ultrasound, 38(1):4-23, 1997.

Ursic, M.; Ravnik, D.; Hribernik, M.; Pecar, J.; Butinar, J. \& Fazarinc, G. Gross anatomy of the portal vein and hepatic artery ramifications in dogs: corrosion cast study. Anat. Histol. Embryol., 36(2):83-7, 2007.

Wrigley, R. H. Radiographic and ultrasonographic diagnosis of liver diseases in dogs and cats. Vet. Clin. North Am. Small Anim. Pract., 15(1):21-38, 1985.

Dirección para Correspondencia:

V. Gabriela Pidal

Hospital Escuela

Facultad de Ciencias Veterinarias

Universidad de Buenos Aires

Buenos Aires

ARGENTINA

Email: gapidal@fvet.uba.ar

Recibido : 30-06-2014

Aceptado: 07-07-2014 